\title{
ВMJ Global Health Developing a globally applicable evidence-informed competency framework to support capacity strengthening in clinical research
}

\author{
Amélie Julé, ${ }^{1}$ Tamzin Furtado, ${ }^{1}$ Liam Boggs, ${ }^{1}$ Francois van Loggerenberg, ${ }^{1}$ \\ Victoria Ewing, ${ }^{1}$ Manhaz Vahedi, ${ }^{2}$ Pascal Launois, ${ }^{2}$ Trudie Lang ${ }^{1}$
}

To cite: Julé $A$, Furtado $T$ Boggs L, et al. Developing a globally applicable evidenceinformed competency framework to support capacity strengthening in clinical research. BMJ Global Health 2017;2:e000229. doi:10.1136/bmjgh-2016000229

AJ and TF contributed equally.

Received 31 October 2016 Revised 10 February 2017 Accepted 16 February 2017

CrossMark

\footnotetext{
${ }^{1}$ The Global Health Network, Centre for Tropical Medicine and Global Health, University of Oxford, Oxford, UK

${ }^{2}$ Special Programme for Research and Training in Tropical Diseases (TDR), Research capacity Strengthening and Knowledge Management (RCS-KM), World Health Organization, Geneva, Switzerland
}

\section{Correspondence to}

Tamzin Furtado;

tamzin@globalhealthtrials.org

\section{ABSTRACT}

Capacity development for clinical research is held back by a lack of recognition for the skills acquired through involvement in clinical trials and in other varied types of global health research studies. Although some competency frameworks and associated recognised career pathways exist for different clinical research roles, they mostly apply to a single role or study setting. Our experience supports the need for an integrated approach, looking at the many roles in parallel and at all types of clinical research beyond trials. Here, we propose a single, flexible framework which is applicable to the full global health research team, and can be used for recognising staff by highlighting acquired skills and possible progression between various roles. It can also illuminate where capacity needs strengthening and contribute to raising research engagement. Through systematic analysis of existing competency frameworks and current job descriptions covering 11 distinct, broad clinical research roles, we identified and defined 50 key competencies required by the team as a whole and throughout the study life cycle. The competencies are relevant and adaptable to studies that differ in design, geographical location or disease, and fall in five main areas-(1) Ethics, Quality and Risk Management; (2) Study and Site Management; (3) Research Operations; (4) Scientific Thinking; and (5) Professional Skills. A pilot framework and implementation tools are now available online and in paper format. They have the potential to be a new mechanism for enabling research skills development and career progression for all staff engaged in clinical research globally.

\section{INTRODUCTION}

In its 2013 World Health Report (WHR), WHO urged the global research and policy communities to take integrated efforts to strengthen the capacity of all countries to generate evidence for the improvement of their health systems. The need for action towards health research capacity development had already been taken up at the regional level by the Pan American Health

\section{Key questions}

What is already known about this topic?

- Regardless of the size, place and type of clinical research, for a project to be realised, certain steps and tasks remain the same, and must be achieved to a high standard by the clinical research team.

- Those involved in research require adequate, targeted training to acquire the necessary competencies and conduct their role accordingly.

- Competency frameworks help to identify the knowledge, skills and tasks that clinical research staff need to master, but are usually narrow in their focus.

\section{What are the new findings?}

- This study combines existing clinical research frameworks with real-life data to produce a globally applicable framework which is adaptable for different types of research, different global settings, team sizes and disease types.

- Detailed analysis of the desired traits for different clinical research roles highlights that competencies cut across roles.

- The novelty of this work lies in its broad applicability to the research team as a whole, thus highlighting continuity between roles and seeking to recognise all contributions to the clinical research endeavour.

\section{Recommendations for policy}

- This framework can be used in multiple ways, to develop curricula or inform career development for individuals working in clinical research, or to identify gaps in existing research capacity.

- These applications will assist in the delivery of clinical research, while increasing the recognition of all staff for their contribution.

- Career pathways need to be developed to enable all staff to progress in their role as well as in others, in light of their demonstrated competencies rather than their job title.

Organization (PAHO), which first issued a policy document on the matter in 2009. ${ }^{2}$ These two documents particularly emphasise 
the need to develop research capacity in low and middle income countries (LMICs), so that these countries can take ownership in addressing local health issues. Research agendas and practices which are set in highincome settings indeed often fail to adequately target the disease burdens that affect the majority of the world's populations. ${ }^{3}{ }^{4}$ To make real changes to global health outcomes, LMICs must therefore equip themselves with the skills to carry out high-quality research studies that address locally relevant research questions. ${ }^{5}$

The capacity needed to conduct global health studies is multifaceted, and ranges from the funding and physical facilities (ward space, laboratories), through to managerial support and personnel training. ${ }^{5}$ While all building blocks of a well-functioning research system are necessary and interlinked, arguably the key to developing sustainable local capacity comes from having well-trained teams, and individuals who can demonstrate leadership in research, whatever their role. This concept of a capable workforce is central to many capacity development models, which stress the importance of training individuals and ensuring that they are situated within environments of organisational support, mentoring and supervision. ${ }^{2} 67$

Although on the rise, the availability and accessibility of adequate training programmes remain limited for many research staff; ${ }^{9}$ and particularly for those who are not qualified as medical doctors. ${ }^{9}$ Technological developments now make it possible to reach new populations through various models of online training. ${ }^{10-13}$ However, with a lack of understanding of the clinical research skills that need to be developed, it is not clear whether existing 'clinical trials' curricula (whether face-to-face, or online and distance learning) cover the full range of topics to be mastered by global health research staff. In addition, many core roles within clinical research are little understood and still relatively unrecognised as viable career paths. ${ }^{9}{ }^{14}$ This is the feedback we received during discussions with local partners and when we evaluated training exercises we regularly conduct in LMICs, especially on the role of research nurses, trial managers and data staff. ${ }^{815}$

Furthermore, sustainable capacity building must enable research groups to independently plan, obtain funding for and operate high-quality, locally led studies that address most pressing issues. Tackling those require a whole range of clinical research; from epidemiology, to observational studies and cross-sectional surveys, through to interventional trials. When defining a set of competencies for clinical research, we therefore consider that an integrative approach which aims to capture information about a spectrum of clinical research will be most successful and practicable, as it will best help staff determine how to transfer their advanced research skills to different types of project.

Several groups have worked towards clarifying the competencies for specific clinical research roles, and the 'Multi Regional Clinical Trials Center (MRCT) Harmonized Core Competency Framework ${ }^{16-18}$ makes an excellent move towards combining the information about several roles (principal investigator, clinical research coordinator and clinical research associate) into one common set of knowledge domains. Here, we further this integration effort to many more roles, including essential though less recognised ones in more operational or technical positions (eg, laboratory technician, data entry clerk). We also take particular account of LMIC settings.

\section{CREATING THE FRAMEWORK}

To create the Special Programme for Research and Training in Tropical Diseases (TDR) Global Competency Framework for Clinical Research, we looked at the complete set of activities needed for a study to take place, regardless of which individual may perform them. We then examined this information independently of the initial job role it pertained to, to determine the shared competencies (tasks, skills and knowledge) required to support the research endeavour as a whole. The rationale for this approach is that each research project is unique, and the exact distribution of roles and responsibilities within the team are likely to vary depending on the size and demands of the study, as well as on the resources and team available to conduct the trial on the ground. Yet, in any clinical research project there are core, invariant aspects that need to be covered: a protocol must be written, ethics approval must be obtained, data must be collected, verified and analysed, etc. Therefore, the research process and the team undertaking it can be envisioned as a continuum for the definition of cross-cutting 'competency areas'.

This systematic examination and categorisation of clinical research activities involved qualitative content analysis of 28 past competency frameworks and 116 current job descriptions from partners around the world, altogether covering the following 11 broad research roles: data staff (from entry to managerial roles), laboratory scientist (from technician to head of laboratory), trial pharmacist, community engagement staff, research nurse, study physician, principal investigator, trial manager or project coordinator, quality control monitor, ethics review board member and sponsor. The data-driven, evidence-based framework resulting from this work was then appraised by a panel of 20 expert trialists, and accordingly refineddetailed methods are provided in the TDR Global Competency Framework for Clinical Research development report. ${ }^{19}$ In the remainder of this article, we describe the framework (accessible at https://globalhealthtrials.tghn. $\mathrm{org} /$ global-competency-framework-clinical-research/), as well as its associated implementation tools; we share the lessons learnt while developing this global competency framework, and we highlight its potential as a mechanism to strengthen clinical research capacity.

\section{CLINICAL RESEARCH ACTIVITIES REVOLVE AROUND 50 COMPETENCIES}

After several rounds of iterative, thematic categorisation of the textual data that we gathered from multiple 
sources and partners around the world, we found that information from the records included in the analysis crystallised to around 50 distinct competencies, which can be grouped into five balanced categories (figure 1): Ethics, Quality and Risk Management; Study and Site(s) Management; Research Operations; Scientific Thinking; and Professional Skills. The 'Ethics, Quality and Risk Management' area encompasses all the competencies related to putting safeguards into place, so as to ensure the risks to participants and of not reaching a scientific conclusion from the research work are kept as low as possible. The 'Study and Site(s) Management' area focuses on research management, from project coordination through to finances and human resources management, and also includes the ability to communicate to relevant parties; an essential part of large, multinational clinical research. The 'Research Operations' area contains more action-based competencies, and covers most of the practical requirements of interacting with participants, delivering interventions or analysing biological samples to collect data on a daily basis during the study. The 'Scientific Thinking' area concentrates on the planning and interpretation aspects of research, which may require various levels of competencies in key disciplines (eg, medicine, statistics, pharmacology, public health), as well as an understanding of the 'science' of doing research itself and the methods that are applicable to different research questions. Finally, the 'Professional Skills' are generalisable, essential competencies, such as leadership, interpersonal skills or organisational skills.

While the day-to-day tasks and behaviours suggesting competency in the corresponding area will differ between team members and vary according to their specific role, the thorough examination of employers' requirements alongside relevant literature suggests that the underlying competencies are remarkably similar and cross-cutting.

A competency is defined as the knowledge or skill required to carry an activity out, not the activity itself. ${ }^{20}$ Our framework distinguishes between the following three types of competencies: (1) the theory, or knowledge-based competency, which reflects a level of theoretical understanding of a particular topic, and which can be acquired through learning about a field; (2) the capability, or task-based competency, which necessitates the application of technical or practical knowledge to the performance of a specific task, and which is better learnt by doing and (3) the trait, or skillbased competency, which corresponds to the demonstration of appropriate behaviours in various situations, and which is better developed by experiencing and reflecting on the experience.

\section{SIMILARITY BETWEEN COMPETENCIES ALLOWS FOR} A UNIFYING FRAMEWORK

The finalised TDR Global Competency Framework for Clinical Research consists primarily of an encompassing
'Competency Wheel' (figure 1), which is applicable to all research roles. It visually presents the framework with its 50 competencies, distributed into the five categories. The core 'Professional Skills' category is surrounded by the four thematic, clinical research-specific categories (Ethics, Quality and Risk Management; Study and Site(s) Management; Research Operations; Scientific Thinking); and the latter are each further divided into 3-4 areas of competencies. While figure 1 provides a paper version of the framework, an interactive version allows navigating it more easily and is available online (https://globalhealthtrials. tghn.org/competencywheel/).

A 'Competency Dictionary' has been created as a direct companion to the Competency Wheel, and provides detail on what is meant by each of the 50 competencies (figure 2). The Competency Dictionary thus explains the overall, generic framework by suggesting a definition, further clarified with keywords, for each competency. Essential skills and knowledge required to master the competency are also listed along with the competencies that are related to it and that appear elsewhere in the framework. Usual abilities associated with the competency are given and directly derived from analysed documents; without those illustrative examples being job specific. The full Competency Dictionary is downloadable at https://globalhealthtrials.tghn.org/site media/media/medialibrary/2016/11/TDR_Framework_ Competency_Dictionary.pdf.

\section{THE FRAMEWORK CAN FACILITATE TRAINING AND CAREER DEVELOPIMENT}

The core, unifying wheel and the dictionary are supported by practical implementation tools, particularly meant to support assessment and follow-up of an individual's competencies.

Drawing on previously developed guidance on scoring and evaluating one's skill level, ${ }^{22}$ a 'Competency Radar' (figure 3) has been developed, and can be used to grade individuals on each of the areas of competency defined within the TDR Global Competency Framework for Clinical Research. Consistent grading should be used as much as possible, in line with the scale developed for the Professional Membership Scheme (PMS),${ }^{22}$ and to facilitate comparisons of competency levels, over time and between individuals. In the future, this consistent grading process will be further facilitated through the release of role-specific frameworks mapped to the generic one, to provide more detailed, targeted examples of tasks and expected levels for each role and competency.

Once filled in, the Competency Radar (figure 3) readily shows the areas of strengths of the concerned individual based on their past experience, and outlines which of their skills could be transferred to a different study or setting. It is indeed important to consider one role's activities within the global picture of competencies, so as to highlight the significance of their 
Design \& planning of research

- Health-related knowledge

- Research methodology

- Developing a protocol

- Attracting funding

\section{Protocol operationalization}

- Developing study plans and documents

- Developing the QMS and SOPs

- Developing the CRF and DMS

\section{Interpretation of study results}

- Analysing data

- Disseminating research findings tudy results

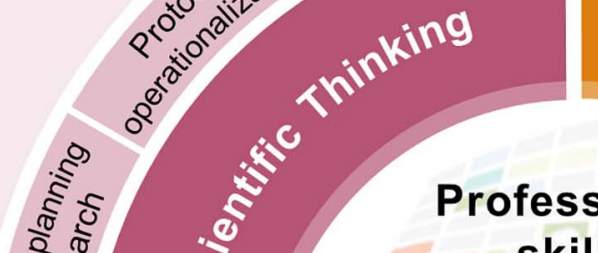
skills

- Cognitive skills

- Strategic leadership

- Interpersonal skills

- Language \& communication

- Organisational skills

- Record-keeping

- Computer \& IT skills

- Work ethic

Oversight

- Initiating study

- Closing study

- Project management

- Tracking study progress

Study communications

- Reporting

- Liaising or acting as a link

- Facilitating or attending meetings

Staff management

- Human resources

- Creating or delivering training

- Supervising or mentoring

Resources management

- Overseeing essential documents

- Logistics and facilities management

- Finances management
Safeguards

- Ethics and human subject protection

- Risk and safety management

Determining liability and insurance needs

Quality assurance

- Good Clinical (or other) Practice

- Working as per the QMS

- Controlling quality of research

Regulations \& governance

- Securing or maintaining approvals

- Securing or maintaining contracts

- Governance and organisational context

- Research regulations

Figure 1 The Competency Wheel or one-page overview of the framework. All 50 competencies are visible in this overall representation of the framework. CRF, Case Record Form; DMS, Data Management System; IMPs, Investigational Medical Products; IT, Information Technology (computing); QMS, Quality Management System; SOPs, Standard Operating Procedures. 
Figure 2 Annotated sample 'Competency Dictionary' entry. The 'Supervising or mentoring' competency from the Study and Site(s) Management area of competency is used as an example to illustrate how competencies have been defined. listed above have its specific entry in the companion dictionary, which can be downloaded at https://globalhealthtrials.tghn.org/ site_media/media/medialibrary/ 2016/11/TDR_Framework_ Competency_Dictionary.pdf. Each of the 50 competencies

Title of dictionary entry, providing the name of the competency being explained

\begin{tabular}{|c|c|c|}
\hline Type of the competency; & Competency & Supervising or mentoring \\
\hline knowledge, skill or task & Type & Task-based \\
\hline $\begin{array}{r}\text { Definition, corresponding to } \\
\text { the synthetic description } \\
\text { derived from the analysis of } \\
\text { varied text data } \\
\text { What other competencies } \\
\text { within the TDR Framework } \\
\text { are related to that one, and } \\
\text { likely to be mastered by an }\end{array}$ & Definition & $\begin{array}{l}\text { The individual has the ability to lead, motivate, mentor, supervise, monitor, train and advise } \\
\text { staff in their work, acting as an expert resource on a specific topic if necessary. They } \\
\text { supervise and line-manage staff, conducting appraisals when needed, and have skills in } \\
\text { motivating and guiding their team. They can assign work appropriately depending on the } \\
\text { interests and skills of individuals, so as to maximise the effectiveness of their team. They can } \\
\text { oversee the activities of other staff members, identifying knowledge gaps and encouraging } \\
\text { training if needed. The individual understands the different needs and management styles } \\
\text { available and adapt appropriately to less experienced staff. } \\
\text { Supervision and mentorship requires good interpersonal skills, the ability to give clear } \\
\text { instructions and feedback, and project management skills so as to assign tasks appropriately. }\end{array}$ \\
\hline $\begin{array}{l}\text { individual proficient in the } \\
\text { competency here described }\end{array}$ & $\begin{array}{l}\text { Related } \\
\text { competencies }\end{array}$ & $\begin{array}{l}\text { Similar tasks: Project management; Human resources; Creating or delivering training } \\
\text { Required skills: Strategic leadership; Interpersonal skills }\end{array}$ \\
\hline $\begin{array}{l}\text { List of words that are } \\
\text { evocative of the competency }\end{array}$ & Keywords & $\begin{array}{l}\text { Supervision; Mentorship; Knowledgeable resource; Support; Advising; Line-managing; } \\
\text { Appraising; Appraisal }\end{array}$ \\
\hline $\begin{array}{r}\text { Examples of abilities or } \\
\text { behaviours demonstrated } \\
\text { by the proficient individual } \\
\text { (non role specific) }\end{array}$ & Abilities & $\begin{array}{l}\text { - Support and guide other researchers or a team and monitor their performance, } \\
\text { developing their skills and capacity as needed } \\
\text { - Mentor new staff; act as an technical advisor or expert to staff or researchers } \\
\text { - Help and support other researchers (whether with work or personal issues, learning, } \\
\text { etc.); provide comprehensive advice and guidance } \\
\text { - Be aware of the various styles of supervision, and understand the principles of sound } \\
\text { supervision/motivationtechniques and their applications in the work environment } \\
\text { - Line-manage and conduct appraisals for staff } \\
\text { - Supervise and coordinate the work of the team } \\
\text { - Evaluate and assign work/delegate to others, based on an individual's strengths }\end{array}$ \\
\hline
\end{tabular}

Figure 3 Example of completed 'Competency Radar'. The grading scheme accompanying the Special Programme for Research and Training in Tropical Diseases (TDR) Global Competency Framework was carefully applied to assess the competency level of a junior researcher in global health. The radar shape readily highlights areas of strengths for this young investigator (who has been involved in designing, planning and interpreting study results), as opposed to major areas where further training and experience should be sought, in line with career plans (eg, the present researcher still lacks confidence in the performance of managerial tasks). The image was generated using the online App for the Competency Radar.

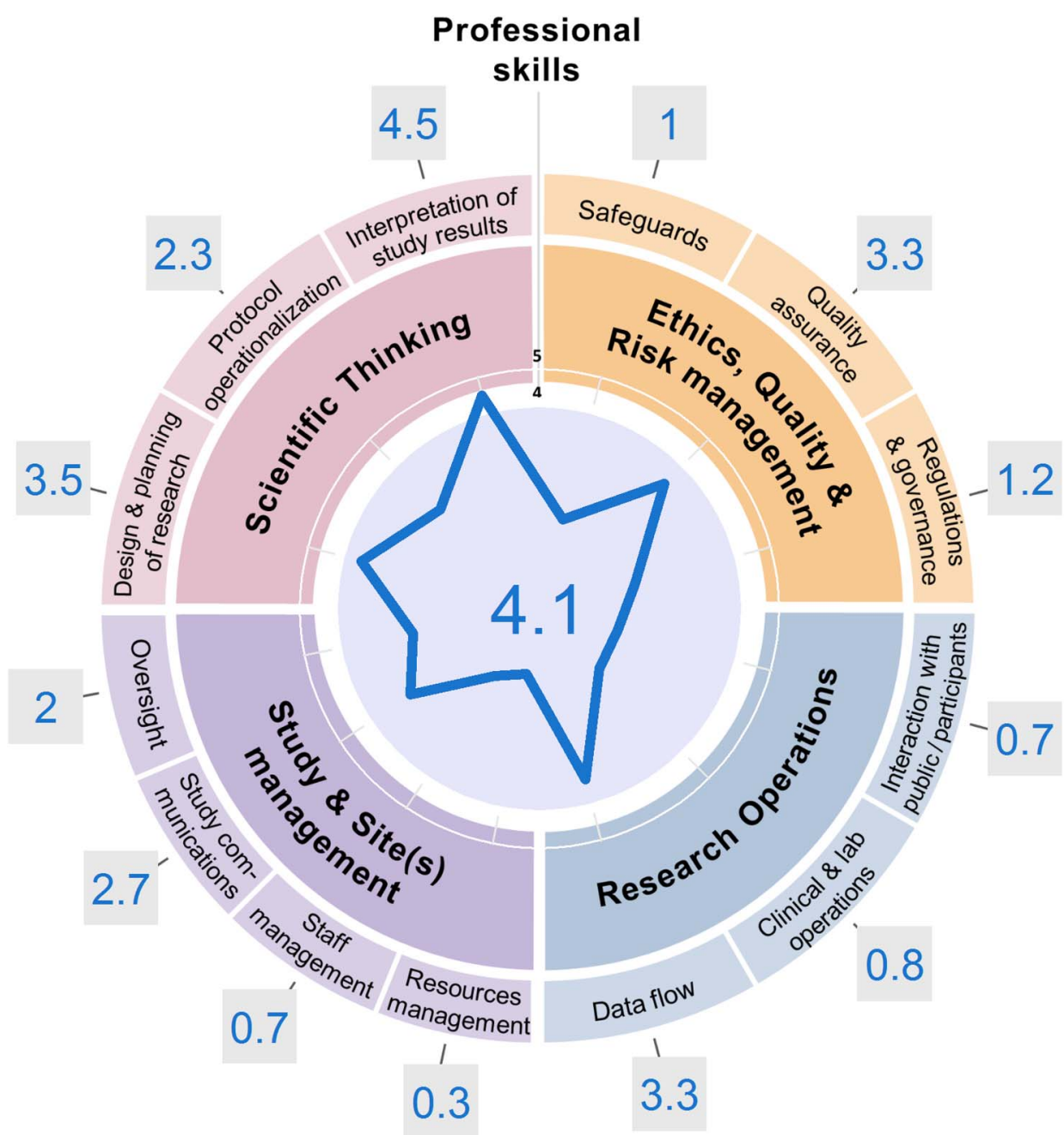


contribution to the study, and their possible career pathways. Past experience of TDR and The Global Health Network suggests there is demand for such a flexible and endorsed system, so as to assist individuals with their career progression. The $\mathrm{PMS}^{22}$ on which the present grading scheme is derived was initially built by The Global Health Network and TDR as a means of providing a free, standardised system for trialists of all roles to track and demonstrate their progression as they build their careers in research; and it currently counts 2500 members as of February 2017.

As a complementary supporting tool, a 'Piloting Protocol' (available at https://globalhealthtrials.tghn. org/site_media/media/medialibrary/2016/11/TDR_ Framework_User_Guide.pdf) has been developed to provide further guidance about how to pilot test and use the framework in practice. This protocol also contains a feedback questionnaire, which the early adopters are encouraged to use to let the authors know of their experience using this beta version.

\section{FURTHER DEVELOPMENT TO REINFORCE THE FRAMEWORK}

While the framework is designed to evolve as we learn, it is currently being released in a beta version, a necessary step to facilitate its further development and testing. Continuous evaluation is indeed envisioned, so as to iteratively improve and validate the work and tools in line with users' needs. This learning process should offset the limitations discussed below.

First, job descriptions have been used as an easily accessible means of determining the roles, tasks and ideal attributes of applicants. These provide a list of the activities each individual undertakes, the training basis required for that role, and which key skills or personal attributes are desirable or essential. However, we recognise there could be a discrepancy in what a job description describes and the actual day-to-day tasks undertaken by an individual. This problem is likely to have limited impact on the overall framework's picture, but we recommend additional research and different methods to further the development of role-specific frameworks. This will be particularly needed for roles where we had limited access to job descriptions, such as the role of the study sponsor, which is critical to clarify to enable locally led research in LMICs.

Second, our framework aims to cover all types of clinical research and thus job descriptions from many diverse locations and trial types were taken into consideration. While this provides the widest possible range of competencies and highlights the connections between apparently different roles, it also means that some of the tasks and roles will not be applicable to every situation. Similarly, we strived to derive broadly applicable and adaptable competency definitions; these may, however, appear too generic in some cases. The generic picture of competencies is nonetheless beneficial to promote career development, as it enables to emphasise bridges between roles and to assess the skills and experience research staff have acquired in a comparable manner. The adoption of this whole-encompassing view is anticipated to lead to better career recognition and capacity building, provided that there is institutional buy-in with creation of adequate training and job opportunities on the ground.

In line with Lucia and Lepsinger's recommendations for the validation of competency frameworks, ${ }^{21}$ the pilot testing will seek to: (1) show face validity, by ensuring that individuals who are currently working in each role understand the competencies suggested, and how they apply to their role; (2) determine whether key tasks have been identified and are clearly covered within one competency or another; (3) determine what the "predictors of success' are for each role, that is, which behaviour demonstrates that the individual has mastered a competency.

Data from this testing phase will first serve to clarify how users interpret the proposed definitions, to address the face-validity question. A feedback questionnaire has been developed, allowing for in-depth comments on the easiness of understanding of different competencies. Where necessary, changes will be made to the framework or the wording of competencies and/or their definition, to improve the comprehensiveness of the framework.

Data from different users of the framework will also enlighten the key skills and tasks that all job roles require for the successful conduct of a study, including but beyond the investigator. We therefore encourage research teams to adopt and pilot test this framework to capture whether competencies are present or missing at the individual and at the larger, site level. Research teams should benefit from using the framework, as their progress can be tracked and demonstrated over time using the proposed tools; hence their efforts can be better directed towards addressing most pressing training or recruitment needs.

\section{CONCLUSION}

The Global Health Network and TDR have created a unifying framework which makes use of pre-existing, more role-specific or setting-specific frameworks, as well as of real-life data. The TDR Global Competency Framework for clinical research is broadly applicable and has been successfully appraised by multiple experts; in addition, stakeholders based in LMICs have particularly emphasised the need for such a scheme. In light of this support, the TDR framework has been launched along with its tools to enable refining this resource in the future, based on users' experience of applying it in context. This framework is laying the ground for future work in the field of clinical research capacity strengthening; and we encourage teams to get involved and use this scheme. Their feedback will be very valuable in refining and widely implementing this valuable new tool for research capacity development. 
Handling editor Seye Abimbola.

Acknowledgements The authors would like to thank the following individuals for their reviews and feedback on the framework during the workshop on the development of a global core competency framework for clinical research held at the WHO head quarter, Geneva, Switzerland on 29-30 September 2015: Barbara E. Bierer, Christian Burri, Núria Casamitjana, C. Padma Chandrasekaran, Roma Chilengi, Helen Demarest, Michäel Kaeser, David Lalloo, Kamal Mansinho, Renata Mendizabal Sole de Cabrera, Wilfried Mutombo Kalonji, Jean Nachega, Raffaella Ravinetto, Morven Roberts, Nidia Rizzo, Nandi Siegfried, Nathalie Strub Wourgaft, Alfred Tiono, Jeremy Whitty. The authors would also like to thank Elizabeth Allen and Cordelia Reddy of the University of Cape Town, and Barbara Farrell of the UK Trial Managers Network, for their invaluable support and feedback in the initial stages of development. We also thank Alexander Mietke, Martin Hadley and Alexis Sergt for support with developing the online version of the framework.

Contributors All authors contributed to designing the study. TF and AJ collected and analysed the data with particular support and comments from VE and FVL. AJ created the design of the framework and its online App. TF and AJ wrote the first draft. FVL, VE, TL, LB, MV and PL reviewed and approved the manuscript. TL is the guarantor of the work.

Funding The Special Programme for Research and Training in Tropical Diseases (TDR).

Competing interests All authors have completed the ICMJE uniform disclosure form at http://www.icmje.org/coi_disclosure.pdf and declare: financial support for the submitted work from TDR; The Global Health Network is funded by the Bill and Melinda Gates Foundation; AJ holds a Nuffield Department of Medicine (NDM, University of Oxford) Prize Studentship, jointly covered by the Medical Research Council (UK) and NDM; no other relationships or activities that could appear to have influenced the submitted work.

Provenance and peer review Not commissioned; externally peer reviewed.

Data sharing statement The study brought together existing data, mainly publically available data, which are referenced in the online reports, and from data obtained upon request and subject from a number of different sources. Full details how these data were obtained are available in the documentation available at https://globalhealthtrials.tghn.org/articles/tdr-global-competencyframework-clinical-research-set-tools-help-develop-clinical-researchers/.

Open Access This is an Open Access article distributed in accordance with the Creative Commons Attribution Non Commercial (CC BY-NC 4.0) license, which permits others to distribute, remix, adapt, build upon this work noncommercially, and license their derivative works on different terms, provided the original work is properly cited and the use is non-commercial. See: http:// creativecommons.org/licenses/by-nc/4.0/

\section{REFERENCES}

1. World Health Organization. The World Health Report 2013: Research for universal health coverage. Luxembourg: WHO Press, 2013.

2. Pan American Health Organization/World Health Organization (PAHO/WHO). PAHO's Policy on Research for Health: 49th Directing Council, 61st Session of the Regional Committee
(CD49/10). 10 July 2009. http://www2.paho.org/hq/index.php? option=com_content\&view=article\&id=1414\%3A2009-policy-onresearch-health\&catid $=2502 \%$.

3. Yozwiak NL, Happi CT, Grant DS, et al. Roots, not parachutes: research collaborations combat outbreaks. Cell 2016;166:5-8.

4. Miranda JJ, Zaman MJ. Exporting "failure": why research from rich countries may not benefit the developing world. Rev saúde pública 2010;44:185-9.

5. Franzen SRP, Chandler C, Lang T. Health research capacity development in low and middle income countries: reality or rhetoric? A systematic meta-narrative review of the qualitative literature. BMJ Open 2017;7:e012332.

6. Potter C, Brough R. Systemic capacity building: a hierarchy of needs. Heal Policy Plan. Vol. 19. Cardiff, UK: Department of Epidemiology, Statistics and Community Medicine, University of Wales College of Medicine, 2004:336-45.

7. ESSENCE on Health Research. Seven principles for strengthening research capacity in low- and middle-income countries: simple ideas in a complex world. World Health Organization, 2014.

8. Franzen SRP, Chandler C, Enquselassie F, et al. Understanding the investigators: a qualitative study investigating the barriers and enablers to the implementation of local investigator-initiated clinical trials in Ethiopia. BMJ Open 2013;3:e003616.

9. Lang TA, White NJ, Tran HT, et al. Clinical research in resource-limited settings: enhancing research capacity and working together to make trials less complicated. PLoS Negl Trop Dis 2010;4:4-7.

10. The Global Health Network. The Global Health Network: enabling research by sharing knowledge. 2015 (cited 7 August 2015). https:// tghn.org/

11. Coursera Inc. Coursera. 2015. https://www.coursera.org/.

12. edX Inc. edX [Internet] (cited 15 January 2015). https://www.edx.org/

13. London School of Hygiene and Tropical Medicine. Masters in Clinical Trials (Distance Learning) (cited 15 January 2015). http:// www.lshtm.ac.uk/study/masters/dmsct.html

14. Ajay S, Bhatt A. Training needs of clinical research associates. Perspect Clin Res Clinical Operations, Feasibility and Site ID, Quintiles India, 5th Floor, Leela Business Park, M. V. Road, Andheri (E), Mumbai-400 059, India. 2010;1:134-8.

15. Furtado T, Franzen S, van Loggerenberg F, et al. Strengthening neglected tropical disease research through enhancing research-site capacity: an evaluation of a novel web application to facilitate research collaborations. PLoS Negl Trop Dis 2014;8:e3225.

16. Multi-Regional Clinical Trials Initiative. MRCT Resources. 2016 (cited 10 January 2016). http://mrctcenter.org/resources/

17. Thomas Jones C, Browning S, Gladson B, et al. Defining competencies in clinical research: Issues in clinical research education. Res Pract 2012;3:99-107.

18. Sonstein SA, Seltzer J, Li R, et al. Moving From Compliance to Competency: A Harmonized Core Competency Framework for the Clinical Research Professional. Clin Res 2014:17-23.

19. WHO/TDR. Development of the TDR global competency framework for clinical research. Geneva: World Health Organization, 2016.

20. Royal College of Nursing Research Society. Research Nurse Competency Framework-version 2. 2011. https://www2.rcn.org. uk/_data/assets/pdf_file/0019/201466/Research_Nurse Competency_Framework_-_Version_2_-_Full_-_Oct_2011.pdf.

21. Lucia $\mathrm{AD}$, Lepsinger $\mathrm{R}$. The art and science of competency models: pinpointing critical success factors in organizations. San Francisco: John Wiley \& Sons, Inc., 1999.

22. TDR, The Global Health Network. Global Health Network Professional Membership Scheme. 2015 (cited 15 January 2015). https://globalhealthtrainingcentre.tghn.org/cpd/ 


\section{Correction: Developing a globally applicable evidence- informed competency framework to support capacity strengthening in clinical research}

Julé A, Furtado T, Boggs L, et al. Developing a globally applicable evidenceinformed competency framework to support capacity strengthening in clinical research. BMJ Global Health 2017;2:e000229.

In the competing interests section the following statement: 'The Global Health Network is funded by the Bill and Melinda Gates Foundation' incorrectly infers that the Bill \& Melinda Gates foundation is the sole funder. This is inaccurate and should read: 'The Global Health Network is funded in part by a grant from the Bill \& Melinda Gates Foundation.'

Open Access This is an Open Access article distributed in accordance with the Creative Commons Attribution Non Commercial (CC BY-NC 4.0) license, which permits others to distribute, remix, adapt, build upon this work non-commercially, and license their derivative works on different terms, provided the original work is properly cited and the use is non-commercial. See: http://creativecommons.org/licenses/by-nc/4.0/

(C) Article author(s) (or their employer(s) unless otherwise stated in the text of the article) 2017. All rights reserved. No commercial use is permitted unless otherwise expressly granted.

BMJ Glob Health 2017;2:e000229corr1. doi:10.1136/bmjgh-2016-000229corr1

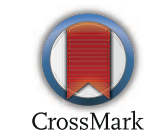

